\title{
Longitudinal associations of skipping breakfast with ethnicity and cardiometabolic risk: the Determinants of Adolescence, now young Adults, Social well-being and Health Study (DASH)
}

\author{
L.M. Goff ${ }^{1}$, M.J. Silva ${ }^{1}$, C. Bordoli ${ }^{1}$, Z.E. Enayat ${ }^{1}$, A. Cassidy ${ }^{1}$, M. Maynard ${ }^{2}$ and S. Harding $^{1}$ \\ ${ }^{1}$ Division of Diabetes \& Nutritional Sciences, King's College London, UK and \\ ${ }^{2}$ School of Health \& Well-Being, Leeds Beckett University, UK
}

Ethnic inequalities in cardiometabolic disease ${ }^{(1,2)}$ may be explained by differences in diet and lifestyle. Poor dietary habits, such as skipping breakfast and consumption of fizzy drinks and fast foods are more common amongst ethnic minority children and adolescents $^{(3,4)}$. The long-term effects of these childhood behaviours on adult cardiometabolic risk factors have not yet been investigated in an ethnically diverse population. We aimed to assess ethnic patterns in adolescent and young adult breakfast skipping and its influence on cardiometabolic risk in young adulthood amongst a diverse UK cohort.

The DASH cohort was recruited in 2002/03 and consisted of 6643 11-13 year olds, sampled to represent the main ethnic groups of the UK population. The 'DASH 10 years on' study is a longitudinal follow-up of a subset of the cohort who are now young adults (21-23 years). Participants had anthropometric measures (weight, BMI, waist circumference), blood pressure, total and HDL-cholesterol and HbAlc assessed and completed a short dietary behaviours questionnaire indicating how frequently they consume breakfast (daily, 3-4 days a week, 1-2 days a week, never/hardly ever).

The cohort consisted of 311 males (age 22.8 (95\% CI 22.7, 22.9) years; BMI $24.7(95 \%$ CI 24.3, 25.2) kg/m²) and 316 females (age $22.7(95 \%$ CI 22.6, 22.8) years; BMI $24.9(95 \%$ CI 24.3, 25.5) kg/m²). A total of 107 White British, 102 Black Caribbean, 132 Black African, 99 Indian, 111 Bangladeshi or Pakistani and 115 Other (mainly mixed) were included in the follow-up. In young adulthood regular breakfast skipping was reported by $56 \%$ of participants; Black African participants were more likely to skip breakfast than White British (OR: 1.81, 1.04 to 3.17, $p=0.004$ ). The highest proportion of breakfast skipping occurred amongst the Black Caribbean $(66 \%)$ and Black African (64\%) groups and the lowest amongst Indian participants (46\%). The impact of skipping breakfast during both adolescence and young adulthood on cardiometabolic risk factors during young adulthood were investigated using multivariate regression modelling. Skipping breakfast at 11-13 years was a significant determinant of BMI at 21-23 years (1.45 (95\% CI 0.61, $2 \cdot 29), p=0.001)$ as was skipping breakfast at 21-23 years, although the effect was slightly attenuated in this age group (0.92 (95\% CI $0 \cdot 1,1 \cdot 73), p=0 \cdot 027)$. Skipping breakfast at both 11-13 years and 21-23 years were also important determinants of total cholesterol levels $(11-13$ years: 0.17 (95\% CI 0.01, 0.33), $p=0.041 ; 21-23$ years: 0.23 (95\% CI 0.07, 0.38), $p=0.003)$.

This is the first longitudinal assessment of breakfast skipping and its impact on cardiometabolic risk factors amongst an ethnically diverse cohort of young adults in the UK. In this work we have recognised the detrimental impact of childhood breakfast skipping on cardiometabolic risk factors, such as BMI and cholesterol concentrations, in young adulthood. Furthermore we have identified distinct ethnic patterns in breakfast skipping, such that skipping breakfast is most prevalent amongst Black African and Caribbean groups and less common amongst Indians. Our findings provide a useful insight into dietary behaviours that health promotion campaigns could target in aiming to improve the diets of young people, and highlights the importance of targeting interventions to improve dietary behaviours such as breakfast consumption at specific groups of young adults in the population.

1. Becker E et al. (2006) National Centre for Social Research.

2. Zhang Q et al. (2009) Ethnicity \& Health 14(5): 439-57.

3. Harding S et al. (2008) Int J Epi 37(1): 162-72.

4. Nicklas TA et al. (1998) J Am Diet Assoc 98(12): 1432-8. 